\title{
II. Kanzlei- u. Archivwesen der frankischen Hohenzollern von Mitte des 15. bis zur Mitte des 16. Jahrhunderts. Von
}

Dr. F. Wagner, Gymnasiallehrer in Berlin.

\section{Standort der Archive.}

Bei den fränkischen Hohenzollern begann ein geordnetes Kanzlei- und Archivwesen 1) sicherlich schon zu den Zeiten Friedrichs I., des eigentlichen Begründers der Macht seines Hanses. Fs sind zwar nur einige zerstreute Notizen erhalten, welche darauf hindeuten, aber sie reichen doch aus, um zu erkennen, welcher Werth auf die Erhaltung und Verwahrung der Urkunden gelegt wurde. In seinem 1437 verfassten Theilungsvertrage verordnete dieser umsichtige Fürst2): „es sollen ausser den Heiligthümern, goldnen und silbernen Gefüssen u. a. die Bücher auf dem Schlosse Plassenburg und dem Behältniss daselbst ewig und unverückt bleiben". Es kann kaum einem Zweifel unterliegen, dass unter diesem Behältniss das später so häufig erwähnte "Gewölbe“ oder „Briefgewölbe“ zu verstehen ist. - Jedoch bezog sich diese Verordnung nur auf die fränkischen Archivalien. Ueber die brandenburgischen findet sich in demselben Aktenstücke die ausdrückliche Bestimmung, dass alle „gemein briefe, zu unsern landen der marck gehorende“, in Tangermünde aufbewalırt werden sollen, weil sie dort sicherer lägen als in irgend einem anderen Schlosse. Wenn ein anderer hohenzollern. scher Fürst ihrer bedürfe, so sollen sie ihm ausgeantwortet werden, jedoch unter der Bedingung, dass er sie nach genommener Einsicht unverzüiglich und ungefäbrdet wieder nach Tangermünde in die

1) Vgl. den Aufaatz: Schicksale des Plassenburger Archivs im 9. Bande dieser Zeitschrift. S. 77 ff.

2) Riedel Cod. dipl. Brandenburgensis III. 1. S. 23 n. 
"Behältnisse" abliefere. Von Wichtigkeit ist auch noch die generelle Bestimmung, dass alle Lehenbücher, Register und Schuldbücher abgeschrieben und jedem der vier Söhne, unter die der Kurfürst seine Lünder theilte, eine Abschrift gegeben werde. Diese Sorgfalt wird nicht in Erstaunen setzen bei einen Fürsten, der in sein Hausgesetz die Bestimmung aufnahm: „unsere deutsche lespuchere, die wollen wir bei unserem leben, ob got will, an ein stat oder closter orden und schicken, da sie alweg bleiben und da nicht vorrucket werden sollen; doch welicher der obgnanten unser sune einer oder mer die abeschreiben wolten lassen, den sol man $d y$ leihen, doch das er vor ein gewissheit tue, sullich pucher in einer benannten zeit wider an sullich stat oder closter zu schicken und zII antworten, dahin wir die orden und schicken werden."

Eine gewisse archivalische Nomenclatur ist in dieser Zeit auch schon erkennbar; die erwähnten Lehenbücher, Schuldbücher, Register, sowie ein in späterer Zeit zuweilen erwähntes „Landbuch"1) und ein „alt buech“ deuten darauf hin, dass bereits Zusammenstellungen der sachlich zusammengehörigen Stücke gemacht worden sind, während der Ausdruck „briefe“ jedenfalls die einzeln aufbewahrten Originalien bezeichnet.

Wenn nun aber auch in der erwähnten Disposition ein Ge sammtarchiv in Plassenburg für die frünkischen Lünder eingerichtet wurde, so besagt jenes Instrument doch auch ausdrücklich, dass die besonderen Briefe, welcho sich blos auf das Unterland oder blos auf das Oberland beziehen, in Verwahrung desjenigen Fürsten, der über einen dieser Theile herrsche, bleiben mögen nach seiner Nothdurft und nach seinem Gutdünken.

Unmittelbar an diese Verordnung Friedrichs I. schliesst sich die berühmte dispositio Achillea seines Sohnes Albrecht vom Jahre 1473 an. In dieser heisst es ausdrücklich: „so sullen alle privilegia, bullen, hantvesten und ander brive, die zu dem land zu Francken gehören, zu Cadoltzpurg und die so zu dem gebirg gehörn, zu Plassemberg ligen und verwaret sein“.2) - Allerdings findet sich keine Notiz, die darauf hindeutete, dass in den Zeiten des Kurfürsten

1) Lang: Nenere Geschichte des Fürstenthums Baireuth S. 48 giebt an, dass ein Landbuch uber die Herrschaft Plassenburg vom J. 1398 und über Baireuth ein solches von 1398 und 1444 vorhanden sei.

2) Riedel Cod. dipl. Brandenburgensis III. 2. S. 82. 
selbst oder seiner Nachfolger diese Anordnung genau befolgt worden wäre; vielmehr kam es ganz von selbst dahin, dass die Archivalien am Sitz der Regierung d. h. in Onolzbach oder Ansbach für das Unterland aufbewahıt wurden. Dass es bei Lebzeiten Albrechts selbst nicht so gehalten worden ist, zeigt eine Notiz aus einem Briefe desselben an die Räthe in Ansbach vom 12. Dezember $1471^{1}$ ), wo es heisst . . . . ,darumb so begern wir an ench mit ernst, das ir den Völcker darnach suchen und uns alle pullen und privelegia, die wir von allen bebsten, davon so vil der sein, und auch der pullen allen, darin wir erlaubnus haben, wo interdicta sind und gehalten werden, das man vor uns celebriren mog etc. abschrift und copeyen sunderlich herein gein Berlin schicken wollet .... ob aber dieselben pullen oder der aller abschrift nicht zu Onoltzpach weren, sundern bey andern privelegien zu Blassemberg, das es dann zum aller furderlichsten zu gescheen hinauf geschriben und bestellt werde" etc.

Man könnte einwenden, dass es sich bei dieser Recherche mehr um die Registratur in Ansbacb, um kurrente Aktenstïcke gehandelt habe. Aber es herrscht einige Jahrzehnte spater noch derselbe Zustand und gerade die erwăhnte dispositio Achillea vom Jahre 1473 kann um das J. 1530 doch kaum noch zu den tăglich im Gebrauch verwendeten Geschăftsakten gerechnet werden. Und doch findet sich im Nürnberger Kreisarchiv hinter einer Abschrift dieses wichtigen Dokumentes die Notiz von der Hand des damaligen Kanzlers Georg Vogler: das original ligt in der schachtel, daran geschriben steet: testamentbrieff marggrave Friedrichs des eltern im 1440. Item marggrave Albrechts taylung zwischen $\mathrm{s}$. g. sonen mit I signirt in gewelb zu Onolzbach. Und wieder 10 Jahre spăter heisst es in einem (auch in Nürnb. A. befindlichen) Briefe des Hauptmanns auf dem Gebirge Wolf von Schaumberg und des Landschreibers Johann Kindlein (Montag nach Lätare 1540) an den Markgrafen Georg den Frommen: der gewünchte Theilungsbrief befănde sich nicht in der „Registratur" zu Plassenburg, aber er könne ja im Gewölbe zu Onolzbach liegen, möglicherweise sei er auch in die Mark Brandenburg gekommen. Es scheint auch insofern ein Unterschied zwischen der Regisiratur und dem Archiv răumlich bestanden zu haben, als es einmal heisst (im J. 1539), „das original ligt etc. in dem behelter

') Burkhardt: das funfft merckisch Buch S. 6. 7. 
des klainen canzleistubleinso der gemechleins", 1) während sonst immer vom gewelb oder briefgewelb in Onolzbach the Rede ist. Belege dafür, dass nicht blos auf der Plassenburg, sondern - und noch reichhaltiger als das dortige - auch in Ansbach ein Archiv vorhanden gewesen ist, werden sich im weiteren Verlaufe der Abhandlung noch mehrfach ergeben.

\section{Die Beamten in Ansbach.}

a) Der Kanzler.

Im Unterlande stand an der Spitze der Kanzlei und des Archivs der Kanzler. Lange Zeit waren Geistliche ausschliesslich zu diesem Amte berufen worden. Erst unter dem Kurfürsten Albrecht findet sich der gelehrte Ritter Georg von Absberg seit 1460 im Besitz dieser Würde. Aber schon damals war seine rechte Hand der Sekretarius Johann Volker, der unmittelbar nach dem Tode des Kurfürsten unter dessen Söhnen Friedrich und Sigismund selbst zum Kanzler aufstieg, wahhrend Georg von Absberg Landhofmeister wurde. Erst mit Volker wurde, so zu sagen, ein technisch ansgebildeter Beamter, der auch von unten auf gedient und welchem Kurfürst Albrecht bereits volles Vertrauen geschenkt batte, zur Leitung der Kanzlei und des Archirs, das er allerdings schon als Sekretarius verwaltet haben mag, ${ }^{2}$ ) berufen. Am anschaulichsten wird seine Stellung und Verantwortlichkeit gekennzeichnet durch den Eid, den er im Jahre 1486 ableisten musste und dessen Wortlaut uns erhalten ist. Er lautet:

„Hernach volgt des canzlers eyd, den HannsVölcker gesch worn hat.

1) Als bei der Abreise des Kurfiursten Albrecht in die Mark 1471 ein Inventarium im Residenzschlosse zu Ansbach aufgenommen wurde, fand sich in der Kanzlei: 1 peth, 3 polsterlein, 1 deck, 2 par leilach, 1 leilach ist zurissen, 2 peth, 1 gestraimpte deck. - Riedel cod. dipl. Br. III. 2. S. 47. Diese Ausstattung scheint auf 3 ständige Beamte hinzudeuten. Dazn wüde auch die Notiz stimmen (bei Lang Neuere Gesch. d. Furstenth. Bayreuth I. 18), dass die Hofordnung des Kurfarsten Albrecht Achilles 3 Schreiber vorgesehen habe, wahrend die Hofordnung seines Sohnes Friedrich vom J. 1509 den Bestand der Kanzlei festsetzte auf 3 Sekretarien, 1 Rentmeister, 1 Kammerschreiber, 1 Gegenschreiber, 1 Substituten, 1 Gerichtschreiber, 4 Kanzleischreiber, 1 Kanzleiknecht, den Dr. Pfotel mit seinem Knaben (Kopisten?).

2) Einige Belege dafür in den Forschungen zur deutschen Geschichte. XXIV. p. 477. 
Item das er der canzlei und was darinnen zu handeln nottorftig ist getreulich mit allem vileis nach seinem besten vermogen und hochster verstentnus vor sein und in allen sachen vleissigs aufsehen haben soll, damit meinen gn. herren in den lehenbuechern, schuldbuechern, registern und in allen briven, previlegien und missiven nichts verseumbt noch einicherlei beschedigung zugefuegt werde, dadurch der herschaft entziehung, schaden oder unrat entsteen mocht, sonder das alles getreulichen verwaren und besonder mit den sigeln m. gn. h. getreulichen umbzugeen, die zu verwaren und in solcher acht $z \mathrm{u}$ haben und zu halten, zu bestellen, das durch ine noch nyements nichts damit versigelt werd oder ausgee, es geschee dann mit wissen und gehais $\mathbf{m}$. gn. h. oder in irer gnaden abwesen durch rate, den derselben ibrer guaden sach zu handeln bevolhen wurdet; mit den er auch, so er dabei ist und sunst fur sich selbst alzeit in allen sachen unser gn. h. und der herschaft nutzes und bestes getreulichen brufen, suchen, handeln, furnemen und raten helfen; auch den rate und alle gehein, so durch ine und vor im in der canzlei und sunst durch schrift und wort gebandelt wurt und sonderlich auch aller brive und previlegia inhalt und ausweisung, die die herschaft hat, denselben iren gn. und der herschaft zu schaden nicht offenbaren, sagen oder melden woll in kain weis oder wege, sonder das alles und iedes bis in sein tod verschweigen und alles das thon, das einem getreuen rat und canzler in seinem stat, handel und furnemen geburt, sonder alle arglist und genzlich ongeverde. Also helf mir got und die hailigen". - Hinter dieser Eidesformel steht die Bemerkung: „hat nachvolgend ain andern aid than meinem gn. $h$. marggrave Fridrichen allain (d. b. also nach Markgraf Sigismunds Tode, der in J. 1495 erfolgte), als der hernach registrirt ist". Wesentliche Aenderungen hat dieser später wirklich noch einınal verzeichnete Eid nicht erfahren; es ist nur die Versicherung am Ende hinzugekommen, ohne Wissen des Markgrafen keinem andern Fürsten dienen $z u$ wollen, etwaige Lehenspflichten ausgenommen.

Volker bekleideto das Kanzleramt bis zu seinem Tode, der 1504 erfolgt sein mag. Einige Jahre hindurch tritt dann kein Kanzler auf; erst 1509 scheint Dr. Lorcher dazu ernannt worden zu sein. Noch später wird Vugler oberster Sekretarius und Vicekanzler, endlich Kanzler. Und 1536 erhält Sebastian Heller diese Würde. 
b) Der Jundschreiber unter dem Gebirge.

Unter dem Kanzler stand zunächst der Iandschreiber, der zugleich (ierichtsschreiber am Kaiserlichen Landgericht zu Nürnbergr war. Diese Stellung bekleidete lange Zeit Georg Gruber; häufig tritt er von 1486-1500 in den Akten auf. Für ilın hat sich das Eidformular nicht erhalten; doch ist dieser Verlust nicht bedeutend, da es in wesentlichen übereingestimmt lıaben wird mit dem des Landschreibers auf dem Gebirge, das weiter unten mitgetheilt werden soll. In Beziehung auf seine Stellung als Gerichtsschreiber heisst es:

„Hernachvolgt des gerichtschreibers eide, den Jorg Gruber gescliworen liat.

Ich glub und schwer baiden meinen gn. herrn und der herschafft getreu und gewer zu sein, iren frommen zu furdern, schaden zu waren und ire sachen, so mir bevolhen wurdt, zu ieder zeit getreulich und nach meinem besten verstentnus zu handeln, auch der gericht getreulich und vleissig $z u$ warten, die handlung doran mit vleis aufzuzaichen und was von briven, kuntschaften oder urkunden darein bracht wurdt, getreulichen zu verwaren und was von den urtailern in gesprech gehandelt wurt, den partheien oder iemants von iren wegen nit zu eroffnen, ee dann die urtail ausgesagt werden, und zum rechten einer ieden parthei, die doran zu handeln hat, gleich und unpartheisch zu sein, auch alle und igliche unser gn. l. und der herschaft gehaim bis in mein todt zu verschweigen, alles getreulichen und ongeverde".

Unter diesem Eidschwur steht die Bemerkung: Hermannus Schnat hat disen gerichtsschreiber eydt mitsambt den artickeln, so vil dits lantgericht berurt, auf heut donnerstag nach dem pfingstag [3. Juni] globt und gesworn. Actum ut s. im XVo und ersten. - Nach der Wiederaufrichtung des Landgerichts 1490 wurde der Fid noch anders formulirt. Da lautete er: lantschreibers aide Georg Gruber geschworn das ich soll und will des lantgerichts recht und gewonheit nach ordnung der reformacion oder ferrerm beschaid m. gn. h. getreulich helfen hanthaben, des lantgerichts bucher in vleissiger hut oder verwarung halten, auch dorein oder doraus nichts geverlichs schreiben oder tillgen, all spruch, auch die urtailn von den urtailern an lantgericht furpracht und gesprochen, getreulich uffzaichen und einschreiben und mit namen in des lantgericht bücher nichts schreiben oder schreiben heissen, das sich 
nicht geburt von lantgerichtswegen, auch das einschreiben also getreulich tun und das niemants zu schaden oder zu frumen mindern oder meren, mich auch in keinen urteilbrive, der geverlich radiert oder dorin der gerichtstag oder datum nit geschrieben were, noch an kainem ende doran unterschreiben, auch mit niemants an seiner klag oder handlung am lantgericht ansteen, tail oder gmein doran haben, die urtailn am lantgericht, ehe die ausgesprochen werden von den urtailern, heimlich halten und verschweigen und dem armen als dem reichen, einem als dem andern ongeverlich zum rechten gleich und gmein sein und in dem allen nit anders geverlich thun oder lassen weder durch schenk, myet, gab, freuntschaft, veindschaft, lieb oder laide, noch durch einiche ander sachen in kein weis; das ich auch des lantgerichts sigl alle und igliche fleissig verwarn und getreulich damit umbgeen will und nichts versigeln, das sich von lantgerichts wegen nit gepuret und das gelt, so von den briefen gefellt und mir in meiner bestellung nit zugestellt ist, einnemen, getreulich ufschreiben, m. gn. h. verrechnen und irn gnaden oder wem sie das zu beschaiden geben nach irer gn. bevelh und dorin on irer gn. willen nimants nichts nachlassen an dem, das in der reformacion fur die brief zu geben ufgesetzt ist $\mathrm{m}$. gn. h. zugehorig oder durch den lantrichter aus bevelh $\mathrm{m}$. gn. h. ferner taxiert oder geordent wurd alles getreulich und ongeverlich. Also helff mir got und die heiligen".

Jedenfalls hatte der Landschreiber unter der Oberaufsicht des Kanzlers die Verwaltung des Archivs zu leiten. Doch scheint es fast, als ob ihm der Zutritt ohne Zuziehung eines andern Beamten nicht freigestanden habe. Wenigstens schreibt der spätere Landschreiber (auf dem Gebirge allerdings) Friedrich Prucker an den Markgrafen Kasimir - undatirtes Konzept im Archive des germanischen Museums; wohl in's Jahr 1520 zu setzen - : „so waiss e. g., dass mir e. g. vater die slussel zu gewelb, auch ein slussel zu des Volkers (des oben erwähnten Kanzlers) ledlin befohlen hat; hab ich die sach bisher seinem befelh nach gehalten, dass ich allein nit in das gewelb gangen bin ......"

Ueber die amtliche Thätigkeit Grubers in Kanzlei- und Archivsachen lässt sich nicht viel ermitteln, theils weil die unermüdliche Arbeitskraft des Kanzlers Volker alle ihm untergebenen Beamten weit in den Schatten stellte, theils weil er offters als Gesandter und Orator gebraucht worden ist. So z. B. noch auf dem Reichs- 
tare zu Augsburg im Jahre 1500. Bald nachher muss er in Ungnade gefallen sein; denn an 23. November 1501 erwiedert der Markgraf auf ein Anschreiben der Räthe in Ansbach, das Benchmen und die Worte des alten Landschreibers Georg Gruber hätten ihn sehr verdriesslich und unwillig gemacht, da diesem ron seinem Vater und ihm so viel Gnade bewiesen worden sei; 1 ) doch infulge seines unterthänigen Ansuchens und der Fürbitte der Räthe wolle er der gütllichen Verhandlung beistimmen. Ueber die Ursache des Streits hat sich nichts auffinden lassen. Dagegen wirft folgende Notiz einiges Licht auf dessen Beendigung:

„Hannsen von Eybs hofmeisters handtschrift der letzten abred zwischen $\mathrm{m}$. gn. hern und Jorg Grubern

Jorg Gruber hat meinem gn. h. marggrave Fridrichen zugesagt, s. g. von haus aus $z u$ dienen in $s$. g. selbs sachen vor meniglich und wider meniglich getreulich und ungeverlich, also das Jorg Gruber s. g. in vertrauen und $\mathrm{m}$. gn. h. wider in sein vertrauen gestelt haben. Daruff hat im $\mathrm{m}$. gn. herr 50 gulden soldt, hofclaider, futer und cost, so er ine s. g. dinst erfordert, zugesagt. Daruff hat Jorg s. g. auf die alten pflicht glubd getan'“.2)

Diese Notiz ist zwar undatirt; sie steht aber unmittelbar vor ciner solchen aus dem Jabre 1503. Die Stelle als Landschreiber war aber schon am 14. Dezember 1590 neu besetzt worden mit Endres Gattenhover, der seit dem 6. März 1491 Hofgerichtsschreiber gewesen war. Als solcher hatte er den vorgeschriebenen Eid an

1) In dieser Beziehung ist folgender Eintrag lehrreich: „Am donerstag vor S. Johannis sunwenden [22. Juni] im 97. hat $m$. gn. h. marggraf Friderich Jorgen Gruber landschreiber bevolhen sein lehenbrief zu übergeben der lehen, die Sebastian Beutelbach wiltmaister zu Kreulsheim sel. verlassen hat nach laut der notell, die s. g. gehort und bewilligt hat und hat s. g. dazumol in beiwesen Ulrich von Zedwitz hofmeister und Johann Volkers Kanzlers gesagt: auf dein zusagen, das du dein lẹbenlang bei mir und meinen sonen pleiben wollest, lass ich solches gescheen. Hat der landtschreiber geantwurt: das will ich thon, so mir kain ungnad erzaigt wurd".

2) An diese Notiz uber die materielle Stellung möchte ich eine - leider undatirte - Petition anschliessen folgenden Inhalts :

„Gunstiger lieber herr haubtmann, wir bitten euch, ir wollet furdern, dass zu den 25 gulden deputats noch jedes jars jegklichem ain halbfuder weins gegeben werd. Das ist dannocht dem nit gleich, wie es vormals gehalten worden ist. Dann vor 20,25 jaren hat man geben 20 gulden an gelt, jegklicher person ain fuder weins und darzu ain anzal korns.

Cautzley, Landschreiber und Gerichtschreiber". 
Wagner :

dem genannten Tage dem Kanzler Volker und das Gelïbde dem Landschreiber Geory (iruber als Substituten des Landgerichts gethan. - Ihm folgte 1504 Johamn Tettelbach 1), der im J. 1486 Kanzleischreiber, bez. Registrator, 1493 Sekretarius in der niederländischen, 1501 in der oberländischen Kanzlei und 1503 Privatsekretarius des Markgrafen Kasimir geworden war. Wir werden ilhn später in seiner Thätigkeit als Landschreiber noch näher kennen lernen.

\section{c) Die Sekretarien.}

Unter dem Kanzler und Landschreiber stand ein ziemlich zahlreiches Personal. Zunächst kamen die Sekretarien, deren Eidformulare aus dem Jahre 1486 auch noch rorhanden sind:

„Hiernach volgt Liuhart Nordlingers eyd zu der canzlei und sunst, den auch, so ferr es die canzlei beruert, Hans Vogell und Veit Henlin als secretarien geschworn haben.

Ich glob und schwer baiden meinen gn. hern, marggrave Friderich und marggrave Signund, das ich in der canzlei und was darinnen zu handeln ist, getreulich mit allem vleis nach meinen besten vermogen und hochsten verstentnus handeln und vleissigs aufsehen haben soll und will, damit meinen gn. hern in den leheibuchern, schuldbuchern, registern und in allen briven, previlegien und missiven nichts versenmet noch einicherlei beschedigung zugefugt werd, dadurch der hersehaft entziehung, schad oder unrate entsteen mocht, sondern das alles dem canzler getreulich helfen verwarn und handeln und besonder mit den sigeln meiner gn. hern, so mir der je zu zeiten eins oder mer von iren gnaden odler irem canzler befolhen wurden, getreulich umbzugehen, die zu verwaren und in solcher acht zu haben und zu halten, das nichts damit versigelt werd oder ausgee, es geschee dann mit wissen und gehais meiner gn. hern oder ires canzlers oder in abwesen irer gnaden und des canzlers durch rate irer gnaden rete, den derselben irer gnaden sach bevolhen wurt, mit den ich auch, so ich dabei bin, und sunst fur mich selbs alzeit in allen sachen meiner gn. hern und der herschaft nutz und bests getreulich bruefen, suchen, handeln, furnemen und raten helfen, auch den rat und alle gehaim, so durch mich und vor mir in der canzlei und sunst durch schrift und wort gehandelt wurt und son-

1) Den Eid lejstete er erst am 19. Februar 1505. 
derlich auch aller brive und brevilegia iuhalt und ausweisung, die die herschaft hat, denselben iren gnalen und der herschaft zu schaden, nicht offenbar sagen oder melden will in kain weis oder weg, sonder das alles und jedes bis in mein todt verschweigen soll und will ich getreulich darob und daran sein, damit meiner gn. hern rennt, zins, gult und nutzung zu den rechnungen getreulich verrechet und des nichts verhalten oder entzogen werd, auch nichts von irer gnaden gelt oder anderm, das mir befolhen wurt, ausgeben oder verendern on irer gnaden sonderlich bevelh und wohin durch ir gnad geschafft wurt einich bezalung zu thun, dagegen von einem jeden geburlich quittantz zu nemen und in alweg mit irer gnaden gut getreulichen iren gnaden zum besten nach meiner hochsten verstentnus umbzugeen und $z u$ handeln, auch die rechenbuecher und register getreulichen verwaren und wo ich ichts erfuer irer gnaden schadens, das irn gnaden nit verhalten alles getreulich und ongeverlich. Also helf mir got und die hailigen".

Aus diesem Eidschwur ergiebt sich schon, dass die Sekretarien nicht ausschliesslich in der Kanzlei beschäftigt wurden, sondern auch bei dem Kassen- und Rechnungswesen thätig waren. In der That wissen wir sowohl von Hans Vogel ${ }^{1}$ ) wie auch besonders von dem zuerst genannten Linhard Nordlinger, dass er zugleich Kammerschreiber war, ja, dass er zu militärischen Aufträgen verwendet wurde, insofern diese mit Listen, Registern oder Zetteln zusammen hingen.2) Dass er so auffallend und besonders zuerst genannt wird, könnte auf die Vermuthung führen, dass er der eigentliche Kanzleivorstand gewesen sei. - Auch die Sekretarien wurden oft in Geschäften ausgesandt und hatten deshalb, da sie auch die Fürsten

1) Riedel Cod. dipl. Br. III. 2. p. 244. - Albrecht Aclill sandte ihn 1470 als Kanzleischreiber nach der Mark.

2) Vgl. 35. Jahresbericht des list. Vercins f. Mittelfranken S. 16. Danit hangt auch eine Stelle in einem Bericht des Kanzlers Volker an den Markgrafen Friedrich vom 28. Dezember 1501 zusannmen; der Markgraf hatte seine früheren Ordnungen zur Vertheidigung seines Landes gefordert; der Kanzler uberschickt ihın einige und fährt dann fort: aber wie e. g. die ordnung gemacht hat, do ir auf das lechfelt gezogen seyt, des ist kein verzaichnus bey mir noch in der canzley, sonder Nordlinger sel. mag das bey sein handen gehabt haben und wurd auch bericht, das e. f. g. nach seinem abgang e. g. cammerschreiber Johannes Hofmann in Nordlingers haus geschickt und nach den verzaichnusen und registern suchen lassen und was des funden sey e.g. zu eur selbs gewalt und beheltnus geantwurt. 
im Krieg und Frieden begleiteten, ihre Dienstpferde. Im Unterlande scheinen anfänglich 2 Sekretarien ständig in der Kanzlei verwendet worden zu sein; ausserdem hatte aber jeder der beiden Markgrafen noch seinen Privatsekretarius; aber selbst die Markgräfin hatte noch einen solchen zu ihrer Verfügung. Sie rekrutirten sich unzweifelhaft aus den Kanzleischreibern und rückten bei Vakanzen in die Stellung des Landschreibers oder Kanzlers ein. Zu den angeseheneren Beamten gehörten sie unzweifelhaft, da bei Testamenten, Staatsverträgen, Berathungen ihre Anwesenheit ausdrücklich erwähnt wird. Ueber ihr Einkommen vermag ich keine sichere Angabe zu machen; doch waren sie nicht blos auf ein fixirtes Gehalt angewiesen, sondern hatten aus Sporteln und Accidenzien eine Vermehrung desselben zu erwarten, wie sich dies aus nachstehendem Briefe des Sekretarius Christoph Klaus in Ansbach an den Sekretarius auf dem Gebirge Johann Tettelbach vom Sonntag Dorothea 1502 (6. Februar) klärlich ergiebt:

„Mein her cantzler hat uns allen hieniden in der cantzlei gesagt, das es vormals mit den birgischen cantzleigefel gehalten worden, also das dasselb alles herabgefolgt sei ausserhalben lehen und bekantnus und was ander brieve in des haubtmans verwaltung und namen ungeverlich ausgangen sein. Das hat der lantschreiber eingenomen, als wir auch vermerken, das es her Hainrich Joss mit demselbigen .... ordenlich gehalten, er hab auch ein slussel zu der buchsen oder dem tisch gehabt. Aber als wir in der cantzlei itzo hienyden bericht werden, so soll es ytzo ein ander weis haben, euch und uns allen zu nachteil und schaden. Versele ich mich, das es eur mainung je nit sein solt; dann solt des birgisch gefell nichts und das gefell hieniden wenig sein (als es auch ist), habt ir zu bedenken, das unser aller tail wird schmal sein. Deshalben bit ich euch von gemeiner cantzlei wegen, ir wollet selbs darein sehen, damit uns nichts entzogen, auch kein neuerung gemacht werd. Last mich auch wissen, wievil des gefels ytzo daoben gefallen sey; dann es will mein notturft erfordern, damit ich die taylung darauff wiss zu machen. Und ob euch nit gelegen wer mit dem gefel daoben umbzugeen, so hevelhet es Jorgen Weinperger; er wirt auch on zweifel nichts versäumen. Damit zu guter nacht". 1)

1) Ein hierher gehöriges Schreiben liegt auch vom 27. November 1503 vor. Da schreibt der Kanzleischreiber auf dem Gebirge Hang Rorer an den Sekretarius Cristoff Claus in Ansbach: „Liber her Claus. Uff eur schreiben 
Von Personalien wäre etwa noch zu erwähnen, dass der oben an dritter Stelle erwähnte Veit Henlin ein besonderer Giünstling Markgraf Friedrichs war, ${ }^{1}$ ) wie auch der Markgraf Sigismund seinen Sekretarius Georg Reimlinger noch in seinem Testament erwähnt. Nach dem Tode seines Herrn trat letzterer in die Kanzlei über, die am 11. November 1496 noch Thomas Mendlin und 1498 Christoffel Claus überwiesen erhielt. Am 3. August 1506 leistete Karl Nordlinger den Eid und in Jahre 1509 tritt der spätere Kanzler Georg Vogler als Privatsekretarius des Markgrafen Kasimir auf.

d) Die Registratoren und Kanzleischreiber.

Zu dem untergeordneten Personal gehörten die Registratoren und Kanzleischreiber, für welche das Eidformular 1486 folgendermassen lautete:

„Hernach volgt der gemein schreiber in der canzlei eyd, den Johannes Dettelbach ${ }^{2}$ ) als registrator und Benedic Thalmann ${ }^{3}$ ) und Johannes Scheyr als substitute geschworn haben:

Ich glob und schwer meinen gn. hern getreu und gewer zu sein, iren schaden zu waren, frommen zu werben, in der canzlei und warzu mich ir guad gebrauchen, getreulich zu dienen und irer gnaden gehaim, wo ich die erfar aus schriften, worten oder sunst, in oder ausserhalb der canzlei, zu verschweigen bis in mein todt, auch nichts aus buechern, briven, registern oder schriften abzu-

das ir mir getan habt, der canzlei zu Onolzbach 2 gulden von Hansen Praun zu Culmach und 5 gulden vom pfarrer zur Weissenstat einzufordern und euch zu uberantworten ... hab ich darin vleissig gehandelt und hat mir der pfarrer uff ein ernstlich schrifft, die ich im in der niderlendischen canzlei namen getan hab" etc.

') Im Testamente von 1498 heisst es: „auch sollen meine sön Veiten, meinen secretarien, sein leptag bei in behalten in dem stand, das er sich zu erneren hab und bei in pleiben kan, doch das er ine getreulich und fromklich, als er mir gethon hat, dien .... " Er hat auch neben dem Kanzler Volker an letzter Stelle dieses Testament mitunterschrieben.

?) Er stieg, wie oben schon kemerkt, später zum Sekretär und endlich zum Landschreiber auf.

2) Er war ein Geistlicher und Sohn des ehemaligen Landschreiberg Mathias Thalmann. Der Kanzler Volker verwendete sich Anfang 1487 in einem eigenhündigen Briefe für ihn wegen einer fetten Pfründe, da er noch 3 unerzogene Schwestern und einen Bruder habe; „80 hat ir vater ye getreulich von jugent auf bis in sein tod gedient; dester getroster macht e. g. ander auch getreulich und vleissig zu dienen." 
schreiben oder jemants zu leihen oder zu geben on bevelh irer gnaden, ires canzlers oder der secretarien, die des bevelh oder macht haben und was ich erfuer irer gnaden schadens das z.ı iglicher zeit iren gnaden zu offenbaren und alles das zu thun, das ein getreuer canzelschreiber (sic) und knecht seiner herschaft z.u nutz und frommen thon soll, alles getreulich und ongeverlich; also helf nir got und die hailigen".

Als nach dem Tode des Markgrafen Sigismund 1495 alle Bcamten dem überlebenden Bruder, den Markgrafen Friedrich, ihren Fid erneuern mussten, traten mehrfache Aenderungen ein; so auch bei diesem Formular, das eine noch bestimmtere Fassung erhielt. "Ich glob und schwer, das ich m. gn. h. narggraf Fridrich zu Brandenburg getreu und gewertig sein, s. g. schaden warnen, fromen fordern und s. g. briefe, register und bucher, was mir der abzuschreiben befolhen werden, mit getreuem und ganzem vleis abschreiben und dieselben briefe, register und bucher, auch was ich doraus wurd schreiben, getreulich verwaren, doraus oder davon niemants nichts lesen oder schreiben lassen soll und will on seiner gnad oder die des von $\mathrm{s}$. g. sunderlichen befelh zu jeder zeit haben, sunderlichen wissen und willen, und was ich in solchem oder sunst s. g. oder der hersehaft gehaim erfar niemandts on $\mathbf{s}$. g. befelh will eroffnen, sunder versweigen bis in mein tod, alles getreulich und ongeverlich. Also helf mir got und die heiligen evangelia. Disen aid haben geschworn her Casper Wagner, her Jacob Heffner und Peter Gotz. Actum Onnoltzbach am donnerstag nach dem suntag jubilate (20. April) anno 97. - Johannes Knawr von Rot hat diesen aid auch geschworn am donrstag vor Bonifacii (1. Juni) anno 97. - Sebastian Onolt von Crailshaim hat den obgeschriben aid auch geschworn am dinstag nach Johannis sunwend (27. Juni) anno 97. - Johannes $Z$ winger von Unttern Birnt hat den aid geschworn montag nach misericordias domini (15. April) im 99".

Es scheint übrigens, als wenn 1495 eine strengere Scheidung $\mathbf{z w i s c h e n ~ R e g i s t r a t o r e n ~ u n d ~ b l o s s e n ~ K a n z l e i s c h r e i b e r n ~ e i n g e t r e t e n ~}$ sei und als wenn der zuletzt erwähnte Eid in der späteren Zeit nur für die Registratoren, der obenstehende dagegen nach wie vor für die Kanzleischreiber verwendet worden wäre. Denn hinter dem Eide von 1486. finden sich zahlreiche Einträge auch nach dem Jabre 1495 und bei diesen ist immer ausdrücklich hinzugesetzt, dass der Schwörende den Lid als Kanzleischreiber leiste. So heisst 
es z. B. Jorg Hutter hat disen aid als canzleyschreiber auch geschworn an dinstag nach undecim mil. virg. (23. Okt.) anno 98. - Conntz Gutman hat disen aid als canzleischireiber auch gethan am dinstag nach lichtmes (5. Febr.) anno 99. - Disen aid der canzlei haben auch gethan Bastian Kengel, des thurners sone und Linhart Zechle, des Zechlins thorwarten sone, am montag nach sant Matlieustag apostoli et evangeliste (23. Sept.) anno 99. - Den rorgeschriben der canzley ayd hon ich Cristoff Nesselhauff auch gethan. Actum am montag Calixti (14. Okt.) anno 99. Es folgen noch Johannes Voss, Sebastian Dirolt, Herman Hans Ochsenbach, Hars Pfeil, Karl Ortlen, Johannes Swertfurer, Fritz Pühler, Johannes Hofman, Sebolt Rauft von Rotemburg, Hans Kellner, des Küchenmeisters Sohn, Signund Nurmberger, Christof Gattenhofer, Johannes Merklein" u. a. - Man sieht, dass sich zu diesen Stellen besonders die Söhne der niederen Hofbeamten (Thürmer, Thorwart, Küchenmeister) drängten, wenn sie sich eine etwas bessere Bildung angeeignet hatten. Jedenfalls waren Viele darunter, die sich dem geistlichen Stande gewidmet hatten und in diesen Stellungen die Erlangung einer fetten Pfründe abwarten wollten. - Besonders bemerkenswertb unter diesen kleinen Einträgen wäre etwa noch folgender: „diesen aid hat auch gesworn Karll Entlein, des cantzlers schreiber, dweil er in der canzlei aus und eingeet, das es seinenhalb anch also gehalten werd. Actum Onolzbach am mitwochen nach Johannis baptiste (27. Juni) anno etc. 98."

Gewiss unterschied sich der Registrator in seiner Thätigkeit von den Kanzleischreibern; er wird die eingehenden und abgesendeten Schriftstücke in das Journal eingetragen haben und er wird deswegen auch von vornherein der eigentliche Archivar gewesen sein. Auch lag es ihm sicherlich ob, Kopialbücher, in denen wichtige Aktenstücke aller Art eingeschrieben wurden, herzustellen oder herstellen zu lassen. Die Kunzleischreiber werden zunächst zum Mundiren, seltener zun Expediren der ausgehenden Missiven verwendet, aber auch zum Eintragen in die Kopialbücher herangezogen worden sein. Sicherlich standen sie noch nicht auf der untersten Stufe der Rangordnung, sondern es gab ausser ihnen noch ,junge Gesellen" in der Kanzlei, die erst angelernt wurden. - Es scheint sogar, dass aushilfsweise oder zur Anfertigung bestimmter Arbeiten Hilfskräfte eingestellt worden seien. In dieser Beziehung ist ein Brief des Kurfürsten Albrecht aus dem J. 1476 von Belang. Er 
ist an den Hauptmann auf dem Gebirge Hans von Redwitz gerichtet und lautet: „Lieber getreuer. Wir haben die zwen knecht, so du uns herabgeschickt, angenomen, sie globen und schweren lassen, und ir einem des jahrs 20 gulden zu geben versprochen und so sie in unserm dienst sind, fuetter und cost. Das wolten wir dir also gebandelt nit verhalten, dich darnach von unsern wegen haben zu richten. Und begern an dich mit gantzem ernst, dus du dem knecht, der das salbuch geschreiben soll, in einem jeden ampt dem amptman und castner zugiebst, die ime von unsern wegen zu solchem beraten und beholfen sein, wie negst hie verlassen ist. Und umb den andern knecht, mit dem wollest es auch balten nach laut der hierin verschlossen zetteln und ine, so sich des geburen wirt, umbschicken, ine die erfarung, und was nott sein wird, thun lassen, damit uns die felle zu nutz bracht werden" etc.

\section{Die Beanten auf der Plussenburg. ${ }^{1)}$}

a) Der Hauptmann auf dem Gebirge.

Wie im Unterlande dem Kanzler die Leitung und Verantwortlichkeit für die Kanzlei- und Arehivgeschı̈fte oblag, so war im Oberlande der Hauptmann auf dem Gebirge formell der Chef des Kanzlei- und Archivpersonals. Wir besitzen leider das Eidformular, auf das er sich verpflichten musste, nicht mehr; aber wahrscheinlich würde dies auch in so allgemeinen Ausdrücken abgefasst sein, dass wir daraus keinen Beweis für die oben aufgestellte Behauptung würden ableiten können. Entscheidend dafür ist aber der Umstand, dass aus dem Briefwechsel, der weiter unten mitgetheilt werden soll, ersichtlich wird, wie alle Archivrecherchen und ihre Beantwortung durch die Hand des Hauptmanns gehen, wie er für die Verbesserung der materiellen Lage des Kanzlei- und Archivpersonals in Anspruch genommen wird und sich auch wirklich darum bemüht. Bei dem ausgedehnten Wirkungskreise dieses hohen Beamten ist es ja allerdings selbstrerständlich, dass er nur der ideelle Chef ist, während er

1) Eine kleine Notiz sei hier uber die räumliche Lage der Kanzlei auf der Plassenburg eingeschoben. Markgraf Friedrich hatte 1503 dem Hauptmann auf dem Gebirge befoblen eine Zielstatte (Schiessstand) einzurichten. Darauf antwortete dieger am 25. April. „E. g. baben mir bevolhen e. g. hieoben ein zilstat augserhalb der schlosmauer im graben unter der canzlei oben am wimperg zu Plassemberg . . . . machen zu lassen". 
die wirkliche Leitung dem Landschreiber auf dem Gebirge überliess. 1) Das ergiebt sich z. B. aus einem Schreiben, welches der Hauptmann auf dem Gebirge Conz von Wirsberg dem Markgrafen Friedrich unter dem 20. Oktober 1504 (Archiv des germ. Museums) überschickte: „Gnädiger herr. Alles das, so eur gnad mir jungst der privilegia halb zu Blassemberg geschrieben hat, eur gnad die hinabzuschicken, das soll gescheen, so pald eur gnad lantschreiber, der itzt nit anbeims ist und sunst nymant dartzu komen kan, widerkombt ......"

Die Reihenfolge der hier in Betracht kommenden Hauptleute auf dem Gebirge ist etwa folgende: $1466-75$ Heinrich von Aufsess; 1475 Dietz von der Thann; 1476-83 Hans von Redwitz; von 1483 bis 2. Oktober 1486. Sebastian von Seckendorf (Nolt genannt); von 1486-90 Sigismund von Schwarzenberg; für lange Zeit, etwa bis 1509 Conz von Wirsberg; dann folgt Ludwig von Eyb der jüngere; von 1515-20 Conrad Boss von Flachslanden; dann Hans von Laineck; 1527-28 ist Dr. Christof von Beulwitz Hauptmannsverweser; Fridrich von Lidwach, Wilhelm von Wisenthau und Wolf von Schaumberg. - Die Besoldung des Hauptmanns in der Höhe von 600 Gulden nebst Deputat verpflichtete ihn, dem Landschreiber und den Kanzleibeamten freie Tafel zu gewähren.

\section{b) Der Landschreiber auf dem Gebirge.}

Wenn der Hauptmann auf dem Gebirge eine eigentümlich selbständige Stellung einnahm, so war ihm doch gewissermassen zur Kontrolle der Landschreiber zur Seite gestellt, ohne dessen Mitunterschrift er selten ein Schriftstück abliess. Es entsprach ungefähr dieses Verhältniss dem des Hofmeisters (oder Landhofmeisters) und des Kanzlers im Unterlande. Der eine war eben der ritterliche Repräsentant der Regierungsgewalt, während auf den Schultern des andern die eigentliche Geschäftslast ruhte. Dass er zuweilen sogar Sekretärdienste leistete, beweist das oben schon erwähnte Schreiben Pruckers: „so will ich e. g. nit verhalten, do ich lantschreiber worden, hat man mir ein gemessen teil an geld aus der canzlei jerlich gegeben als einem secretarien. $\mathrm{Nu}$ hab ich bisher weiters nit

1) Wenn Lang Neuere Gesch. I. 33 den gelehrten Rath Dr. Strauss als Chef der oberlandischen Kanzlei bezeichnet, so vermag ich nicht anzugeben, worauf er diese Meinung stützt. 
begert, wiewol ich zu zeiten gresst last der canzlei allein getragen hab. So will ich auch on e. g. willen und rate itzo weiter auch nit begern .... a aber das bit ich itzo undertenigklich, bin ich bei e. g. darfur etwan angesehen zu e. g. und der herschaft .... da dan e. g. ob mir halt, damit ich neben dem lantschreiberambt in der canzlei als ein secretarius pleib und darin mir mein teil nit geschmelert werd" etc. - Die Gehaltsbezüge des Landschreibers bestanden in der freien Tafel am Tische des Hauptmanns, freier Wohnung, der Hofkleidung, der Fourage für 1 Pferd, dem Schreibgelde von den Belehnungen des Adels, $1 / 4$ von dem Handlohn der Kanzleilehen und aller Kanzleisporteln. - Der Landschreiber war ebenso wie der Kanzler Vorsteher der Kanzlei, des Archivs, der Siegelbewahrer, aber freilich auch Finanzbeamter und sogar zur militärischen Bewachung der Plassenburg mitverpflichtet, wie sich dies aus seinem Eide ergiebt:

"Landtschreibers uff dem gebirge eide. Ich glob und schwer den etc. hern, hern Fridrichen und hern Sigmundten, zuvorderst und nach irem abgang (den got gnädiglich langzeit enthalten woll) irer gnaden menlichen erben, die heraus in die landt getsilt sind, getreu und gewertig zu sein, iren frommen zu werben, iren schaden zu warnen, auch irer gnaden nutz getreulich forschung zu haben und die hanthaben belfen nach meinem besten vermogen und ir rent, zins, gult, steur, ungelt, fell und alles ander nach irer gnaden bevelh zu jeder zeit mit einemen und ausgeben getreulich handeln und handeln helfen und so ich ie 200 oder 300 gulden von irer gnaden wegen innen hab, dieselben in das gewelb zu Plassemburg thun und dorin verwarn, auch mit meines gn. hern insigel getreulich umbzugeen, das zu verwaren und in solcher acht zu haben und zu halten, das nichts damit versigelt werde oder ausgee, doran gelegen sei und zu kunftigen zeiten dient, es besche dann mit wissen und gehais meiner gn. hern und sunst in andern sachen mit wissen und bevelh irer gnaden hauptman auf dem gebirg; das ich auch vleissigs aufsehen haben will, damit irer gnaden lehenbuchern, schuldbuchern, reigistern, briven, privilegien und missiven kainerlai beschedigung zugefugt, noch doraus oder daran einicherlai abschrift niments gegeben werde on irer gnaden wissen und willen, sonder nach meinem besten hochsten verstentnus und vermogen, was sich der canzlei halb geburt meinen gn. hern zum 
besten, erlichsten und nutzlichsten handeln und handeln helfen, auch sunst in allen sachen, dabei ich bin, irer gn. nutz und bestes getreulich brufen, suchen, handeln, furnemen und raten helfen, auch den rat und aller irer gn. geheim, die mir zn wissen wurt, verschweigen bis in mein tod; das ich auch des schloss halben das zu verwaren und sunst getreulich will mit helfen zusehen. Ich soll und will auch, dieweil ich irer gn. knecht an dem ampt bin, von kainem, der meinen gn. hern zusteet, noch ainichem andern, der oder die mit iren gnaden oder den iren zu schicken hetten, weder myet, gab oder schanckung nemen, sonder in allen sachen einer parthei sein als der andern und meiner gn. herschafft nutz darin brufen getreulich und ongeverlich."

Dieses wichtige Amt wurde unter der Regierung des Kurfürsten Albrecht Achilles von Hans Imhof, Hartung Rabensteiner und Mathias Thalmann bekleidet. Nach Anton Sattler bekam es Friedrich Prucker, der es von 1483-1518 inne hatte.

Wenn in 9. Bande der Archival. Zeitschr. S. 78 angegeben wird, Friedrich Prucker sei schon 1517 verstorben, so erweist die Unrichtigkeit dieser Notiz ein Brief des Markgrafen Kasimir d. d. Wien 20. März 1518 bei Falckenstein Antiqu. Nordg. vet. IV. 469, worin der alte landschreiber Friederich Pürckler (sic) als noch lebend erwähnt wird. Aus noch späterer Zeit scheint das oben bereits melırfach erwäbnte undatirte Schreiben zu stammen. Ich schliesse das daraus, dass Prucker im J. 1511 von sich sagt, er stehe ob 30 jarn im Dienste der Herrschaft, während er in jenem angiebt, er habe nunmehr der Herrschaft an 41 jar gedient. Von seinem rastlosen Fleisse legt ein Schriftstück Zeugniss ab, das überschrieben ist: „Item nach abgang etc. Albrechts etc. ist durch s. g. son etc. Fridrichen etc. vom anfang s. g. loblichen regirung, die angangen ist zu ostern anno etc. 86 bis uf heut datum diser schrifft s. g. oberlannd uff dem gepirg gebessert durch getreuen rate und vleissige hilff s. g. lantschreibers uff dem gepirg Friderichen Prucker, der sich bisher ob 30 jaren bei der herschafft in diensten getreulich und vleissigklich enthalten hat. Actum uff mitwoch nach reminiscere (19. März) 1500 und eylfften jar." Es schliesst mit den Worten: „Item die obgemelte pesserung $\mathbf{m}$. gn. h. in s. f. g. regirung ufgericht ist angeslagen ob 150000 gulden on die pesserung, die an weiern, zinsten, manschaften noch geschehen mag, also das sich dieselb besserung uff $200000 \mathrm{~g}$. wol erstrecken wirdt. Darumb 
bit der lantschreiber gn. belonung umb sein getreu dienst, die er nu ob 30 jarn bei der herschaft getreulich erzeigt und bewisen hat." Auf Prucker folgte Christoph Clauss und diesem Johannes Kindlein.

c) Die übrigen Beamten.

Auch für das Land auf dem Gebirge gab es natürlich eine vollständige Kanzlei mit Sekretarien, Registratoren und Kanzleischreibern, ganz ebenso wie in Ansbach. Es scheint auch ab und zu eine Versetzung von untergebirgischen Beamten nach dem Oberlande stattgefunden $z u$ haben. So finden wir den oben erwähnten Tettelbach, der doch sonst in Ansbach arbeitete, im Jahre 1502 als Sekretair auf dem Gebirge. Auch die Unterbeamten wurden je nach Bedürfniss bald hier, bald dort verwendet. So ordnet Markgraf Friedrich unter dem 26. August 1501 an, nachdem er das Verzeichniss derjenigen Personen, welche in Ansbach das Deputat erhalten, durchgesehen hat: dass die Räthe „der jungen gesellen ainen in der cantzelei nach der Plassenburg schicken sollen; nachdem uns nit gemaint sein will denselbigen jungen gesellen beden das deputat zu geben". Dagegen remonstrirt der Kanzler allerdings mit Erfolg; denn er erhält am 8. September den neuen Bescheid: da er die beiden jungen Gesellen zur Registratur brauche, solle er sie beide unten behalten; „doch das du solchem deinem anzaigen nach darob seist, damit die hendel, daran gelegen ist, ordenlich werden registrirt."

Als Sekretarieu treten hier auf: Heinz von Joss von 14931501 und ausser Tettelbach noch Hans Zwickstain in den Jahren 1508 und 1509. - Unter den Kanzleischreibern finde ich die Namen: Hieronymus Goller 1500, Hans Renner 1500, Hans Rorer 1503-15, Georg Weinsberger 1502, Georg Arnold, Heinz Plechschmidt und Georg Hofmann 1515.

Eine ganz besonders interessante Persönlichkeit ist die des Registrators Eberhard Frankenberger (oder wie er sich selbst schreibt Erhard Frankenberg), der anfänglich Probst ${ }^{1}$ ) auf der Plassenburg während der Reformationszeit in den Kanzlei- und Archivdienst übernommen wurde. Die schon früher aufgeworfene Frage, ob in

1) Wenn Lang I. 26. bebauptet, der Probst sei schon fruher 2. Archivar (sic) gewesen, so weiss ich nicht, worauf diese Behauptung sich statzt. - Wohl aber hat der Landschreiber auf dem Gebirge Prucker eine Zeit lang neben seinem andern Titel den des Probstes auf der Plassenburg geführt. 
der Person Frankenbergs ein eigentlicher Archivar eingesetzt worden sei, möchte doch nicht so unbedingt zu bejahen sein. Das oben erwähnte Schreiben des Hauptmanns auf dem Gebirge Wolf von Schaumburg und des Landschreibers Johann Kindlein unterstiitzt diese Annahme doch keineswegs. Darnach scheint es vielmehr so, als wenn Requisitionen des Markgrafen nach Archivalien auch im Jahre 1540 noch an diese beiden Beamten gerichtet' worden seien. Und da auch bei anderen Gelegenheiten der Hauptmann auf dem Gebirge Anfragen etwa vorhandene Archivalieu betreffend beantwortet, so ergiebt sich zum mindesten daraus, dass Frankenberg weder direkt mit dem Markgrafen, noch auch mit Statthalter und Räthen verkehrte, sondern, wie es doch früher auch schon gewesen war, unter Verantwortlichkeit des Hauptmanns und Landschreibers das Archiv verwaltete. So lange aber nicht nachgewiesen wird, dass Frankenbergs Stellung ïberhaupt eine neue und eigenartige war, möchte ich nach dem mir vorliegenden Material glauben, dass er nicht mehr war, als viele vor ihm, nämlich Registrator. In dieser Annahme bestärkt mich ein Schreiben des Hauptmanns auf dem Gebirgo Wolf Christoph von Wisenthau an den Markgrafen Georg vom Mittwoch nach Kantate im Jahre 1534, mit welchem dieser dio vom Registrator angefertigten Abschriften der auf Mainbernheim und Heidingsfeld bezüglichen Archivalien (die rogistratur nennt es eine in dorso befindliche Kanzleinotiz) übersendet; zugleich empfiehlt er dem Markgrafen zu gnädiger Berücksichtigung des Regis trat ors Supplikation, da er „eine frume person sei und da im keine vergleichung von der brobstei, so er hievor inngehabt, geschehen sei, sonder er uff eur fürstl. gn. casten allein, wes ime von der registratur wegen gevolg", angewiesen sei. Die Beziehung auf die früher innegehabte Propstei schliesst wohl jeden Zweifel aus, dass dieser hier erwähnte Registrator ein anderer als Erhard Frankenberg sein könnte, zumal da ja auch die Erwähnung der Dürftigkeit, in der er lebt, zu des ebemaligen Propstes Lebensumständen durchaus passt. Nun ergiebt sich aus anderen Archivalien des Nürnberger Archivs allerdings, dass Franckenberg fortwährend darauf hindrängte, die archivalischen Bestände an einem einzigen Orte zu verwahren und die lästige wie zeitraubende Trennung in ein oberländisches Archiv auf der Plassenburg und ein niederländisches im Gewölbe zu Ansbach aufzuheben. Er sagt z. B. item uber diese bieoben gethanen abschrifften, deren originalien 
zu Blassemberg in verwarung sind, werden sonst biemit auch uberschicket: erstlich ein nissive von m. gn. h. marggrave Casimirn ausgangen und dabei 4 sonderliche copeyen von konig Ludwigs zu Hungern etc. derselben originalien aber sind hieoben nit; dieweil aber zuvor in diesen sachen vil und oft gehandelt worden ist nach anzaigung der schriften und widerschriften, deren ains tails hieoben des gebirgs und ains tails daniden sind, so muss man sich daselben auch notturftiglich ersehen, was hierzu dienlichs gefunden werden möchte. Und das doch etwa nach geendter dieser handlung alle originalien mit derselben jetwedern und jegliche zugehorenden briven sei an missiven copeien, instructionen, relationen oder anderm verzeichnisse etc. an ain ort zusammengebracht wurd und hinfuro nicht mehr also in die lenge von einander getailt gelassen blieben, daraus man ein grundtlich wissen haben möchte in der notturft erforderung, was furhanden und was man sich jetweder mals zu gebrauchen durfftig were, dasselbig zu uberschicken ze haben, das ander ligen zu lassen".... Dass Frankenberg das besondere Vertrauen des Markgrafen Georg besessen haben muss, geht daraus hervor, dass er den Auftrag erhielt, sowohl in Ansbach wie auch auf der Plassenburg Repertorien der vorhandenen Archivalien herzustellen.

Uebrigens muss vor dem von Frankenberg 1534 angefertigten Repertorium doch schon ein Nachweis über die Plassenburger Archivalien vorhanden gewesen sein. Denn in einem Schreiben des Markgrafen Georg an den Hauptmann auf dem Gebirge vom 29. Juni 1534 (Nürnb. Arch.) heisst es: „Der Registrator solle, nachdem wir vor etlichen tagen oder wuchen ein verzaichnus aufs gebirg geschickt haben, was in zwaien unsern und der herschaft buchern, mit h. h. und j. j. signirt, registrirt befunden werde, samt seinem Unterricht schleunigst nach Ansbach schicken. Zum andern ist unser mainung, du wollest unserm registrator erustlich bevelhen, das er mit allem getreuen vleis und zum allerfurderlichsten in unserm gewelb und den schachteln darin nachsuchung thue, was allenthalben von brieflichen urkunten in sachen die von Rothenburg an der Tauber berurend gefunden werde und uns davon copien oder registratur ... uberschickt 1 ) ... U Und nachdem wir

1) Jedenfalls entstand daraus das noch heut im Bamberger Archiv vorhandene kleine Repertorium uber die in den Archiven zu Plassenburg und Ansbach vorhandenen Archivalien die Reichsstadt Rothenburg ob der Tauber betreffend vom Jahre 1329-1550. 
in dem Register über das gewelb zu Blassenburg mit oberlendischen sachen unter dem buchstaben $R$ und dem titel Raynung auch noch ein schachtel mit $B$ signirt befinden, darinnen geschrieben steen soll raynung vom Fischsee in den bauchbrunnen" etc.

Aus dem Umstande, dass von dem grossen Inventarium, welches Frankenberg anfertigte, nur ein Band die oberländischen Urkunden, dagegen 4 Tome die niederländischen Stücke umfassen, möchte zu schliessen sein, dass das Ansbacher Archiv damals einen bedeutend grösseren Schatz von Archivalien verwahrt hat als das Plassenburger.

\section{Dor Geschaftsgang.}

Ueber den Geschäftsgang lässt sich aus den zerstreuten Andeutungen noch einiges orschliessen. - Die Konzepte der abzusendenden Schriftstücke wurden entweder vom Kanzler selbst oder von einem der gelehrten Räthe, die zur Bearbeitung der rechtlichen Fragen, zur Berathung in den auswärtigen Angelegenheiten, zur Ausrichtung wichtiger Gesandtschaften am Hofe gehalten wurden, ausnahmsweise auch von den Landschreibern und Sekretarien entworfen. Dabei wurde auf etwa schon vorhandene Schriftstücke stets sorgsam zurückgegriffen. Bei Antworten auf Briefe wurde entweder das Datum des zu beantwortenden Schreibens oder der genaue Inhalt desselben oder wobl auch beides zugleich angegeben. Die Titulaturen bein Eingange und bei der Adresse wurden hierbei nur angedeutet. Die Datirung unterblieb so lange, bis das Konzept genehmigt war. Die Vorlage geschah entweder bei dem Fürsten selbst und darauf mag sich die der kaiserlichen Kanzlei entlehnte Unterschrift, die sich unter dem Kurfürsten Albrecht Achilles zuweilen findet, d. p. se (d. h. dominus per se) beziehen oder die Gutheissung des Konzepts erfolgte in einer Rathsversammlung (dominus in consilio). War dies vollzogen, so erfolgte die Datirung und, falls der Fürst persönlich am Ausstellungsorte sich befand, die Hinzufügung der Stadt oder des Platzes, von dem das Schriftstück ausging. Bei Briefen der Statthalter und Räthe wurde dieser Vermerk neist weggelassen. Eigenhändige Unterschrift des Fürsten ist in dieser Zeit noch ziemlich selten. Das Mundiren, wobei die richtigen Titulaturen einzusetzen oft Schwierigkeiten verursachte, das Siegeln, Adressiren und Abschicken war dann Sache der 
Sekretarien bez. Kanzleischreiber. - Unter die Konzepte wurden oft noch kurze Bemerkungen über Absendung und ähnliche Vermerke gemacht, z. B. wie unserm gn. h. auf annemen des pundts zu Swaben bei Lenhartlin boten geschriben, der ausgeriten ist am donrstag nach Jacobi anno etc. LXXXIII.

Was nun die einlaufenden Schriftstücke anbetrifft, so wurde auf deren Umschlag neben der Adresse zunăchst gewöhnlich ein Präsentationsvermerk gemacht; z. B. durch Lenhartlin boten einbracht am mitwoch nach Kiliani anno etc. IXXXXVIII Oder auf einem Mandat Maximilians rom 11. September 1489 steht: ist Hansen von Wolfstein zu Cadeltzburg geantwort auf heut mitwoch nach exaltationis crucis (16. Sept.) im 89. jare zwischen 11 und 12 horen vormittag. Daraus ergab sich auch, wie viel Zeit der Bote zur Besorgung des Schriftstücks gebraucht hatte. - Sodann wurde auf der Aussenseite eine kurze Inhaltsangabe unter Nennung des Absenders gemacht z. B. von marggraf Johann credentz auf Cristoffel von Aufses gelt berurend. - Darauf folgte eine kurze Verfügung, ob das Schriftstück registrirt werden sollte oder nicht und wohin es zur Aufbewahrung gelegt werden soltte; z. B. pundtsach, in dieselbe laden zu legen. - Sobald die Aktenstücke nicht mehr zum unmittelbaren Gebrauche dienten, wurden sie in Büscheln vereinigt und schon über diese wurde sorgsam Buch geführt. Sehr deutlich ergiebt sich die Genauigkeit des Geschäftsganges bereits in der Zeit des Kurfürsten Albrecht Achilles aus einer von dem oben erwähnten Kanzler Johann Volker eigenhändig angefertigten Registratur, welche sich in einem Sammelbande des Bamberger Kreisarchivs befindet und die folgendermassen lautet:

"I tem der erst buchsel (sic statt buschel) . . . a

${ }^{*} \bar{n}$ credenz auf hern Jobsten vom König . . . . . . . . . a 1

$*_{\bar{n}}$ credenz auf hern Jobsten von hertzog Heinrichen . . . . a 2

${ }^{*} \bar{n}$ abschid hern Jobsten zu Schwabach . . . . . . . . . . a 3

* wie mein her herzog Heinrichen bei hern Jobsten geshrieben hat a 4

${ }^{*} \bar{n}$ wie mein her dem kaiser hern Jobsten handelung geschrieben hat ............... . . . 5

* wie mein her den dechant und Ludwig von Eyb zum kaiser gefertigt und ine gein Dinckelsspuhel nachgeschrieben hat a 6

* wie die rete meinem hern auf dasselb schreiben geantwurt haben a 7 cum una cedula

Die mit * bezeichneten Aktenstucke sind noch jetzt in dem oben bezeichneten Bande vorbanden. 


$$
\text { Item der ander buschel . . . . . b }
$$

das die rete gein Baden einkommen sein . . . . . . . b 1 habet zedulas IV

vom pfalzgraphisch handel mit dem Kaiser

hertzog Albrecht entdeckt herzog Ludwigs handel mit mein hern

wer von fursten zu Baden sei

handel der von Augspurg im stift zu Coln

burgundisch handel

mein her von Mentz contra Beheimen . . . . . . . . . b 2

der dechant von Bamberg, was er Heintz Ruden halb mit

Mentz gehandelt hat . . . . . . . . . b 3

die rete, wie sie durch den kaiser auf ir einbringen gehort sein $b 4$ cum quatuor zedulis

des kaisers antwort in der Beheimischen sach

den kaiser und pfalzgrafen berurend

herzog von Burgundi

bischof von Coln sein capitel

die rete des zusamenkommens halb mit Burgundi . . . . . b 5 zedula una

kaiser und pfalzgraf

lantgraf von Hessen und capitel zu Coln

das des königs von Polan botschaft zum kaiser sei einkommen

die Beheimischen sachen fur den kaiser zu weisen der kaiser des Beheimischen handels halb ... . . . . b 6 wie mein her den reten geantwurt hat . . . . . . b 7

Burgundi

Jorg von Stain

her Jobst meinem hern geschriben.

Polnisch botschaft, die bei dem kaiser ist

wie mein her hern Jobsten geschrieben hat maggraf Friderichs

heirat halb. . . . . . . . . . . . b 8

wie her Jobst meinem hern geantwurt, auch der Beheimischen

sach halb geschriben hat. . . . . . . . . b 9

wie mein her hern Jobsten auf des kaisers briefe geschriben hat b 10 wie die rete geschriben haben der Polnischen botschaft halb,

die im kaiserlichen hofe gewest ist . . . . . . b 11

u. s. w. u. s. w". 
Diese Büschel von Papieren, welche also nicht blos registrirt wurdell, sondern auch ihre Signatur erhielten und meistens die Aktenstücke einer bestimmten Gattung während der Frist eines Jahres umfassten, wurden dann in Schachteln gelegt und sachlich zusammengeordnet. So gab es z. B. Schachteln für die Testamente; die eine wird bezeichnet als diejenige, „daran geschriben stet testamentsbrief marggraf Friderichs des elteren im 1440., item marggraf Albrechts thaylung $z$ wischen s. g. sönen mit 1 signirt in gewelb zu Onolzbach". Vom Testamente des Markgrafen Friedrichs d. Ä. aus dem Jahre 1507 heisst es: „ligt das original in der schachtel mit 2 signirt, darauf geschriben steet marggraf Friderichs testament und kayserliche confirmation darauf gevolgt im gewelb zu Onolzbach". Die verschiedenen Stücke innerhalb jeder Schachtel hatten wieder ihre besondere Signatur. Im allgemeinen mögen die grossen lateinischen Buchstaben als Hauptrubriken rerwendet worden sein, während die Unterabtheilungen mit Ziffern bezeichnet wurden. So heisst es z. B. ums Jahr 1530 in einer archivalischen Recherche: ,item hiebei sonderlich ze wissen, das nach anweisung des gestellten zaigers uber das Onolzbacher gewelbe, nemlich an der schachtl oder ledlin mit A. 3 verzaichent ein vidimus uber konig Sigmundt zu Behaim etc. freiheiten, den von Haidingsfelt und Bernhaim gegeben zu finden ist. Mer daselben im B. 3 brive und handlungen uber Meinbernheim und Haidingsfelt von der behaimischen cron zu lehen rürend die herrn von Guttenstain etc. betreffent ze finden. Auch im B. 4. jüngste handlung zu besehen". Andere Notizen lauten: ,ist dasselb orignal zu Plassenberg im gewelb gelegen in der obgemelten orbainigungs- und andere ainigungsschachtel zwischen Sachsen, Brandenburg und Hessen mit h. h. h. signirt". Oder: „ligt das original auch in der vertragsschachtel mit 3 signirt".

Manche wichtigen Aktenstücke wurden allerdings nicht ohne weiteres in eine beliebige Schachtel gesteckt; sondern dafür gab es besondere "Ledlin" $d$. h. Behältnisse, die manchmal auch versiegelt wurden, zuweilen unter Spezialverschluss des Fürsten sich befanden. So hatte, wie oben erwähnt, der Kanzler Volker eine besondere Lade; so heisst es 1491: „des ist die recht original copei in meines gn. h. marggraf Fridrichs sunderlich beheltnus; so giebt es ein sehr ausführliches verzaichniss der brief in marggrave Johansen sel".1)

1) Er starb als Vizekönig von Valencia. 
ledlein; so verwahrte Markgraf Georg einen vom Herzog Albrecht von Preussen eigenhändig geschriebenen Vertrag besigelt zu Onnolzbach in m. gn. h. marggraf Georgen tisch in s. f. g. camern des neuen thurns. - Schon Ludwig von Eyb sagte in seinen finanziellen Rathschlägen: „der rechnung nimpt im der herr der sum ir jedes ein auszug in sein beheltnus und nimpt der herr ein recess in sein laden .... mit dem hat der herr seins guts und regiments alwegen ein mitwissen". Dass manches Aktenstück auch den Landschreibern und Registratoren unzugänglich gemacht wurde, beweist die Notiz in einem herrschaftlichen Buche des Nürnberger Archivs ... "und ist das original durch den hern canzler Sebastian Hellern und hern Johann Kindlein landtschreibern nach der auscultirung verpetschirt und ins gewelb gein Blassenberg gesetzt ... Michaclis abent $1536^{\prime \prime}$.

\section{Eine Archirrerherche.}

Eine solche Sorgfalt in der Behandlung der Archivalien wurde nicht erst durch die Instruktion des Markgrafen Georg vom J. 1534, als Frankenberg das Inventarium über die Plassenburgische Registratur anfertigen sollte, eingeführt, sondern war schon in den Zeiten des Kurfürsten Albrecht beobachtet worden und wurde durch seinen Eukel nur von neuem eingeschärft. Dafür liefert das Beispiel einer Archivrecherche aus dem Jahre 1532 den besten Beweis. - Markgraf Georg hatte seinen Statthaltern und Räthen befohlen, einen Verzicht auf die Nachfolge in den fränkischen Fürstenthümern, den sein Bruder Albrecht bei der Uebernahme der Hochmeisterwürde des deutschen Ritterordens ausgestellt haben sollte, herbeizuschaffen. Infolgedessen erging an die Landschreiber auf und unter dem Gebirge - dies waren also danals die A rchivdirektoren - der entsprechende Befehl. Aber das Dokument fand sich nicht. Im Plassenburger Archiv veranstaltete Frankenberger (seine eigenhändige Unterschrift: Franckenberg) die Untersuchung und erstattete darüber an den Hauptmann auf dem Gebirge, Friedrich von Lidwach, folgenden Bericht:

„Des hertzogen in Preussen verzigk betreffend.

Mir ist woll in gedenken, das solcher verzig auch vor dreien jaren, als ich dieser zeit in meiner bevolhener arbeithen zu Onnoltzbach ward, begeret, demselben nachgefraget und darumb uffs gebirge geschriben wurd; nicht wayss ich, was dazumal fur antwort gefallen. 
In summa: er ist im gewelbe zu Blassemberg nit, mir auch nie zu handen komen, so doch sonst die allergeringsten (gein dem zu achten) zu merern malen durch mich beschehen und verlesen worden sind, hette ja sich auch dieser nit konnen bergen.

Item so sagt der rentmeister, er hab lang woll gehöret, das ainer vorhanden sein solte, dem zu merern malen nachgefraget worden, er hab sein aber nie gesehen, noch ichts gewises darvon geböret, das er hie oder anderswo sei, wie dann woll darvon geredet worden; sovil und nit mer wiss er davon.

Item so hab ich vor dreien jaren davon oben nicht mer gewisst anzuzeigen dan dis, so ich auch und jetzo hiemit verzaichent dar gebe: nemblich das in dem Onnoltzbacher brieffgewelbe under den schachteln, meiner gn. herrschaft aigne sachen berurnd, aine mit 5 bezaichnet gefunden wurdet, dis thitels oder uberschrift „Bruederlicher vertrag, die statthalterei und jetweders bruders deputat betreffend, item hochmeisters vertrag marggrave Wilhelms halben und des bewilligung darein, auch sein verzigbrief daruber.

Darinnen mag man sich nach notturft besehen

Erhard Franckenberg

probst genant."

Einen noch ausführlicheren Bericht erstattete der Iaandschreiber unter dem Gebirge, Johann Tettelbach, an die Räthe in Ansbach. Darin heisst es: „er habe wiederum das gewelb mit allen schachteln und briefen mit dem besten und hochsten vleis ersucht und ersehen, zusampt dem, das ich herrn Erharden Franckenberger, probst zu Blassemburg, vor etlichen jarn, als er hienyden zu ersuchung der brief im gewelb gewest ist, dazumal auch getreulich hab helfen darnach suchen, aber dazumal, auch jetzo uber allen ganzen moglichen furgewenden vleis ainichen verzig noch ein einichs zettelin dorvon nit finden können noch mogen". Zum Beweise dafür, dass er sich in der von Franckenberg erwähnten Schachtel No. 5 nicht befinde, legt er seinem Bericht einen detaillirten Auszug aus dem erwähnten Behältniss bei.

Auf Grund dieser beiden Berichte erfolgte nun die Antwort der Statthalter und Räthe zu Ansbach an den in Jägerndorf weilenden Markgrafen. Dieser erliess ein höchst bemerkenswerthes Reskript d. d. Jegerndorf montag nach Mathei apostoli 1532.

"Lieben getrenen. Wir haben eur schreiben, das unter und uffm gebirg uff vleissig nachsuchen kein vertzig, den der etc. hertzog 
in Preussen damals, als s. 1. hohemeister in Preussen worden, gegen der herrschaft gethon habe, funden werden kann sambt daneben zugeschickten underrichten alles inhalts vernomen. Wiewol wir nun gentzlich achten, das ein vertzig vorhanden gewest, wo aber derselbig hinkomen sei, wissen wir nit, nachdem wir derselben zeit nit innerlands gewesen sind, so lassen wir es uf dismal bei eurm nachsuchen pleiben. Aber da mogt ir sehen, wie man vor zeiten mit den privilegien und andern briefen und handlungen umbgangen, der jetzt servil, daran auch gross gelegen, in mangel steet, also das nichts registrirt, sonder alle ding unordenlich hin und wider geleget und also derselben weil nit vil gedacht worden, ob ire nachkomen dero notturftig wurden oder nit. Und so man unser jetzige ordnung dagegen hellt, das alle derherrschaft sachen, daran etwas gelegen, mit vleis und ordenlich registrirt und eingeschriben werden sollen, kan ein jetlicher gerings verstands leichtlich mercken was nutz und frumen oder was schaden sich daraus zu vermuten und zu versteen ist. 1) Darumb so wollen wir, sovil uns imer muglich ist, in solchem gute ordnung gehalten haben.

Und nachdem wir wissen, das unser bruder marggraf Casimir sel. mit solchen sachen gar enng (sic) gewesen ist und vil ding zu s. 1. handen zu verwarn genomen hat, so bedencken wir daneben, ob s. l. solchen vertzig etwo bei sich gehabt und s. l. gemahel, unser geschweien, jetzt der hertzogin in Neuburg mit andern dingen mer zu iren handen gestelt hat, den uffzeheben und des ir lieb denselben etwo ungeverlich neben anderm mit irer lieb hinwegk haben mocht.

Ist unser gutbedunken, sover irs anders auch fur gut ansecht, das ir gedachter unser geschweien, unsernhalb unvermerkt oder ungemelt, schreibt und ihrer lieb solchs mit guten glimpf-

1) In dem mir vorliegenden Konzept ist an dieser Stelle der folgende sehr bezeichnende Satz weggestrichen: wiewol die (scil. ordnung) ganz beschwerlich und fur tadelich gehaiten werden will; also ist es vor zeiten zugangen, wir aber wollen, ob gott will, desselben uberig sein. - Ueber die Unordnung welche in der ehemals so vorzuglich eingerichteten Verwaltung der Fürstenthümer Ansbach und Bayreuth nach dem Tode Kasimirs (1527) eingerissen war, und welche derartige Reskripte sehr erklärlich macht, vgl. Voigt Albrecht Alcibiades I. 34. 
lichen worten anzeigt und daneben vermeldet, als ob ir bei uns in grosse ungnad komen, wo ir solchen vertzig nit finden wurdet: ob ir lieb kein wissen davon oder den neben ander irer lieb briefe nit ungeverlicher weis, irer lieb halb unbeweist, mit sich genomen hett ....."

\section{Kopialbueher und Signaturen.}

Während die Originale meist in diesen Schachteln oder Laden aufbewahrt wurden, war man schon von altersher darauf bedacht, von allen wichtigeren Dokumenten sorgfältige und beglaubigte $\mathbf{A b}$ schriften zu nehmen und diese wurden dann in Bücbern vereinigt und dauerhaft eingebunden. Dadurch schonte man nicht nur die Originalien und sicherte sie vor Verschleppung und Vernichtung, sondern gewann auch die Möglichkeit, ein umfangreiches und unentbehrliches Aktenmaterial auf Reichs- und Fürstentage mitnehmen zu können. 1) Auch war es nach der Erwerbung der Mark Brandesburg seitens der Hohenzolleru wünschenswerth geworden, manche Archivalien in dem Archive von Tangermünde, wie in denen auf der Plassenburg und in Ansbach abschriftlich zu besitzen.

Hatte daher schon Kurfürst Friedrich I. im Jahre 1437 verordnet, dass von den Registern und Lehensbüchern Abschriften genommen werden sollten, so scheint unter Albrecht Achilles diese Thätigkeit bereits förmlich systematisch betrieben worden zu sein. Dafür zeugen besonders die kaiserlichen Bücher, welche in sehr dauerhaften Kopialbänden vorliegen. Freilich stammt wohl nur der erste noch aus der Zeit vor dem Tode des Kurfürsten Albrecht; aber der Anstoss, den dieser gewaltige Fürst nach allen Richtungen hin gegeben hatte, wirkte eben noch über sein Leben hinans fort. Von dem zweiten wissen wir ausdrücklich, wer die Abschrift beaufsichtigt und verglichen hat; denn am Ende desselben findet sich die Notiz: anno etc. nonagesimo octavo finivi ego Sebastianus Onolt de Crewlshem hunc librum. ${ }^{2}$ ) Es ist dies unzweifelhaft dieselbe

1) So wurde z. B. ein bedeutendes Material auf den Reichstag nach Worms 1495 mitgenommen. Vom Reichstag zu Augsburg 1500 lässt Markgraf Friedrich nach Ansbach schreiben: item der canzler soll schicken alle handlung in ainer truhen verwart, was er muint $\mathrm{m}$. gn. $h$. hie notturftig werden möcht, als er wol zu bedenken wais. Bei den Archivalien, welche 1536 auf den Tag zu Zerbst mitgenommen wurden, steht die Verfugung, in welliche schachtel sie auch widerumb von dem tag zu Zerbist gethan werden sollen.

2) D. Nahere daraber in d. Zeitschr. f. preuss Gesch. XVIII S. 310. 
Persönlichkeit, welche oben unter den Beamten in Ansbach erwähnt worden ist und aus der Eidesformel der Registratoren rom J. 1495 (s. o.) ergiebt sich ja auch, dass das Abschreiben der Briefe, Register und Bücher zu den Amtsobliegenheiten dieser Beamtenkategorie gehörte. Allerdings beweisen die Schriftzüge, dass der Registrator Onolt von Krailsheim den Text dieses unfangreichen Kopialbuches nicht mit eigener Hand abgeschrieben hat; dazu scheinen vielmehr die ,gemeinen" Schreiber in der Kanzlei verwendet worden zu sein, sondern seine Thätigkeit beschränkte sich darauf, die Abschrift mit den Originalien zu vergleichen, die Fehler zu verbessern, Lücken auszufüllen und an Ende seine Unterschrift in fidem hinzuzufügen. - Während Sebastian Onolt diesen Band im J. 1498 beendigte, hat er im folgenden Jahre ein Eynungs Buch zusammengestellt und unterzeichnet. - In ähnlicher Weise hat der oben erwähnte Johannes Tettelbach als Registrator einen Band videmiert, der die Aufschrift trägt: Anno domini 1400 im 92. jar am suntag nach sant Matheistag ist die ordnung und handelung geschriben aus herrn Ludwig von Eyb rytter buch der herschaffit zu Brandenburg zu gut. Auch hier ist der grösste Theil des Inhalts von einer gleichmässigen gut leserlichen Kanzleihand geschrieben; das Inhaltsverzeichniss, die Ueberschriften und die Schlussworte rühren aber von Tettelbach her. Fr unterzeichnet mit den Worten: Got sey gelobet. I-T d. h. J. T. (Johannes Tettelbach). - Noch früher wurde das Eidbuch zusammengestellt, das mit den Worten beginnt: Anfang dises registers ist gemacht nach abgang m. gn. h. marggrave Albrechts zu Brandburg, curfursten etc., der mit tod verschiden ist zu Franckfort am Mayn am sambstag vor dem suntag judica in der vasten nach Christi geburt vierzehenhundert und im sechsundachtzisten jare, doselbst er auf einem tag was bei kayser Friederichen dem dritten und hern Maximilian etc. etc. Ein anderes Buch umfasst die Kriegsordnungen aus der Zeit Albrecht Achills und seines Sohnes, des Markgrafen Friedrich. Darin findet sich die Notiz: die ordnung des puchs zu schreiben ist angefangen von mir, Contz Cornburger ${ }^{1}$ ) vor Gennt im veld an der mitwoch nach Kiliani [9. Juli] anno 1488 nachmittag; denselben tag war es windig und nass. - Diese Beispiels mögen genügen, um die Thätigkeit der Kanzleibeamten nach der einen Richtung hin zu kennzeichnen.

1) Er leistet am 16. November 1495 den Eid als Kastner von Kadolzburg. 
Uebrigens wurden nicht núr Abschriften in derartige Bücher gebunden, sondern auch die eingegangenen Originalien und die dazu gehörigen Konzepte der abgesendeten Antworten, sobald sich in irgend einer Sache ein umfangreicheres Material angesammelt hatte; nur lässt sich die Zeit, wann dies geschehen sein mag, kaum feststellen. Eine Notiz, die aus der Zeit des Registrators Frankenberg zu stammen scheint, weist schon darauf hin: „nota: ist im ledlein nit funden, sunder ins buch gebruderlicher vertreg mit $T$ signirt gebunden". Die Anzahl dieser Bände ist noch jetzt eine recht bedeutende und wird früher eine sebr ansehnliche gewesen sein. Deshalb wurden diese Tome nicht blos mit einer den Inhalt ungefihr bezeichnenden Aufschrift wie z. B. „Registratur der lehenempfahung, altt und new ainigungs buech, registratur der erb und anderer ainigung, registratur der handlung, so sich durch die schencken von Limpurg gegen marggrafe Albrechten in und mit irer f. gn. glaytt zugetragen, copei der hairat, die ye zu zeiten am hove und durch m. gn. $h$. betaidingt worden sind, abschriften der altreterlichen, veterlichen und brüderlichen vertreg, register etlicher grossen bericht (in vilerlei sachen)", - sondern auch mit einer bestimmten archivalischen Signatur versehen. Ich bin noch im Stande, folgende alte Signaturen nachzuweisen C.C., E.E., F.F., G.G., J., P.P., T., V., T.T., E.E.E., F.F.F. Daraus dürfte sich der Schluss wohl rechtfertigen lassen, dass es allein von solchen, theilweise sehr voluminösen, Bänden mindestens 60 gegehen haben wird. Wahrscheinlich war aber die Zahl noch weit grösser.

Auch die Schachteln oder Lädlein, in denen die Originale verwahrt wurden, hatten, wie oben bereits berührt wurde, ihre Signaturen. So z. B. ledlin A. 3., B., B. 3., B. 4., S. 2. u. s. w.

Ueber den Umfang des Archivs in den verschiedenen Zeitabschnitten Angaben zu machen, ist ungemein schwierig, da bei dem traurigen Schicksal, welches das Plassenburger Archiv betroffen hat, ein nicht unbeträchtlicher Schatz von Urkunden wie von Originalund Kopialbüchern verloren gegangen sein mag. Doch werden die . oben angeführten Inhaltsverzeichnisse und Signaturen, welche später einmal systematisch geordnet werden sollen, schon zur Genüge bewiesen haben, dass selbst der jetzt noch vorhandene Rest sich an Umfang mit den aus dem 15. Jahrhundert stammenden Archivalien anderer Gebiete durchaus messen kann. 
Nachdem diese Arbeit bereits abgeschlossen war, fand ich noch ein Altenstück, das der Mittheilung werth zu sein scheint. Als Georg der Fromme die Regierung der fränkischen Besitzungen 1527 antrat, mochte er schon einen Beamten in seinen Diensten haben, der ein hervorragendes organisatorisches Talent besessen zu haben scheint, nămlich den vielfach erwähnten Kammermeister Leonhard von Gendorf. 1) Dieser verfasste mehrfach Entwürfe zu einer Reorganisation der Verwaltung; besonders hat sich eine ausfübrliche Instruktion erhalten, welche der Markgraf Mittwoch nach Ostern 1531 seinen fränkischen Beamten zur Richtschnur überschickte. Leider wird in derselben nicht die neue Kanzleiordnung selbst mitgetheilt, sondern es heisst darin nur: „was dann sonst der cantzlei halben zu ordnen, das ist in sonderlich verzaichnus bracht und ein abschrifft davon in die cantzlei uberantwort mit des camermaisters handen unterschrieben." Um so schätzbarer ist es, dass sich aus dem J. 1533 ein Protokoll darüber erbalten hat, wie die neue KammerKanzlei- und Hausordnung durchgeführt worden ist. Aus diesem Schriftstück, das vom 3. September 1533 stamnt, möchte ich noch folgende Stellen mittheilen.

„Von gottes gnaden Wir Georg etc. bekennen hiemit für uns selbst etc. als wir aus unsern schlesischen furstenthumben verschiener tagen als nemblich am mitwoch nach Egidii im 33. jar etc. in unsere frenckische landt und erstlich in unser schloss Blassemberg ankomen, haben wir fur uns und dozumal bei uns habende räthe, nemlich Mertein Kanachern, obristen burggraven zu Konsperg, her Hansen von Seckendorf Aberdar ritter, her Sebastian Hellern doctorn cantzler, Wolff Cristoff von Wiesenthau, haubtman ufm gebirg, Jörgen von Benndorff hoffmaister, herr Johann Weynmann doctorn und Leonharten von Jhendorff ${ }^{2}$ ) camermaister etc. unsere neue vor aufgerichte camer-, cantzlei- und hausordnung genomen und darauf aus der niderund oberlendischen camermaisterei und rentmaisterei erfordert wie hernachvolgt ....... (Ich übergehe diesen nicht hierher gehörigen Abschnitt und lasse gleich den zweiten folgen.)

1) Er wird auch im 2. Band der neueren Geschichte des Furstenthums Bayreuth von Lang mebrfach erwhint. schreibt.

9) Das ist der obenerwhhnte Kammermeister, der sich selbst Gendorff 
Den inventarium uber die cantzleien und gewelb betreffendt.

Auf den andern artickel, was der Onnoltzbachischen cantzlei und gewelb inventarium belangt, geben uns unser stathalter diesen bericht, das sovil, in der alten und neuen cantzlei zu Onnoltzbach befunden (ausserhalb etlicher vil schachteln, die im stublein an der alten cantzlei steen), das alles sei in ainen inventarium gebracht, der auch vor der handt und durch Georgen Vogler nach absteen des cantzlerambts in beisein herrn Hannsen von Seckendorff und Sebastian Hellern doctorn und cantzlers, auch Johann Tettelbachs landschreibers aufgericht und mit allen stücken, wie sich gebürt, belegt worden, wie derselbig bei der cantzlei gesuecht und zur notturft gebraucht werden möge; doch also, das solche bücher, in dem inventario begrieffen, den merer thail allein stückweis benannt und darin nit unterschiedlich angezaigt sei, was in ainem jeden buch ordenlich nacheinander stee oder unterschiedlich befunden wurde, das dann ainer sondern, vleissigen arbait und mues (oder mue?) bedarff. Was aber fur schachteln, bücher und handlung im neuen gewelb der silberkamern und dann an schachteln im stublein an der cantzlei, die sein in inventarium nit komen aus ursach, das Georg Vogler die schachteln und handlung im gewelb nit in seinem gewalt gehabt hat. Was dann fur schachteln und handlung in clainem stüblein an der alten cantzlei gewesen, die sind noch vor der handt und möchten mit der zeit erlesen und, was darin ligt, auch in inventarium bracht werden.

Der birgischen cantzlei inventarium ist aufgericht und vorhanden, doch nur stück- oder skatel (sic für schachtel?) weys; aber uber der herrschaft gulden bull, privilegia, freihaiten, brief und anders, so im gewelb zu Blassemburg vorhanden, ist kain inventarium noch verstendig registratur aufgericht.

Darumb haben wir aus erhaischender notturft beschlossen, das ein lauter inventarium uber unser und der herschaft gulden bull, privilegia, freihaiten und anders, so in beden gewelben zu Onnoltzbach und Blassenburg befunden, aufgerichtet werde, nemblich also, das erstlich aller und jegliche brief substantz und datum mit kurtz in ein registratur gezogen, darnach die fürnembsten und genottigsten privilegia und schriften nach ordnung und lengs oder alter der brief in eingebunden bücher abgeschriben, dieselben ab- 
geschribenen bücher sambt den registern, so darüber gemacht, im gewelb bewart werden.

Desgleichen soll es mit den brieven und schriften, so in beden cantzleien unter und aufm gebirg vorhanden gebalten werden, nemblich das erstlich aller und jegklicher brief substantz und datum in ein registratur gezogen, darnach die genottigsten und fürnembsten brief und schriften nach ordnung und lengs in eingebunden bücher eingeschriben und wol bewart werden.

Doch solcher gestalt und mit der beschaidenhait, wann in beden gewelben und cantzleien die notturftigen haubt und andere brief, auch handlung ersehen und aufgeschrieben, das alspaldt darauf die oberlendisch und niderlendisch bücher und registraturn mit vleyss ordenlich nach einander erlesen und ersehen werden, was zuvor darin registrirt befunden, damit zu verhütung $\mathrm{zwifacher}$ arbeit und unnotturftiger uncost allain das, so zuvor nit registrirt befunden würd, vollent registrirt und entweder in dieselben oder andere bücher nach gestalt und gelegenhait der hendel gebracht werden, darin die registratores kunftig der haubtleut, cantzlers, landschreibers und secretarien rath und hilf gebrauchen und jedesmal unterricht empfahen sollen.

Zu solcher aufrichtigung der cantzlei und gewelbinventari, registraturn und abschreibung der fürnembsten und genöttigsten brief und handlungen haben wir unterm gebirg $\mathrm{Hans} \mathrm{Knörn}$, uffm gebirg Erbartenn Brobst geordnet.

\section{Cantzlei ordnung.}

Auch haben wir der cantzlei halben fur notturftig angesehen und beschlossen, das hinfuran alle nöttig und wichtig furfallend sachen, sy betreffen das haus Brandenburg etc. oder die partheyen an, wie die beratschlagt und sovil von nötten für uns gebracht, nach unser oder unser bevelchhaber bewilligung copirt, dieselben copeyen, darnach auch die gestellten schriefften von ainem oder zwaien unser räthe, so bey beratschlagung derselben sachen gewest, abgehort, unterschrieben, durch die registratores einregistrirt und ververtigt werden sollen.

Was dann unser cantzlei ordnung sunsten ober und unterhalb des gebirgs belangt, dorin sollen sich die cantzleien der vorigen inen übergebenen und bevolhnen ordnung gemes halten und erzaigen, bis wir mit vernerm rath in etlichen notwendigen stücken verner einsehen und verordnung thuen." - 
Wie eingehend und verständig diese Kanzleiordnung gewesen sein mag, dafür bieten einige Notizen einen Anhalt, die sich in der allgemeinen Kammer-, Kanzlei- und Hausordnung finden. So z. B. wird über den Einkauf der Kanzleibedürfnisse verordnet:

„Erstlich wöllen wir den extraordinari abstricken, also das furan der rentmaister das papir, birgamen, dintenzeug, wachs, harlaff ${ }^{1)}$ und was auf die cantzlei gehört, selbst bezalen und ist der cantzleiknecht verordnet nymandt nichts davon zu geben, dann weme es zusteet.

Es soll auch der landschreiber hieniden sein birgamen und papir nit mer von landgerichtsgefellen kaufen, sonder alles aus der cantzlei nemen."

In einem späteren Reskript des Markgrafen (Prag, Samstag nach Pfingsten, 25. Mai 1532) hoisst es über die Pflichten des Kauzleiknechtes:

„Und sovil erstlich unser rentmaistereiverwalters und gegenschreibers beschwerungen und anfangs des potenlons halben in der rentmaisterei auszugeben belangt, wollen wir, damit gedachte der rentmaisterei persone irs ambts und bevelhs sonst dester getreulicher und vleissig auswarten mögen, gnediglich bewilligen, das dje teglichen und gemainen pottenlon in der cantzlei, wie etlich zeit vor, durch den cantzleiknecht, wo dieses jetzigen person anderst darzu tuglich und getreu ist, ausgeben, also das demselben wochenlich ain summa gelts vom rentmaister behendigt und herwiderumb alle sein des cantzleiknechts ausgeben in der wuchenrechnung von ime abgehört und aufgenomen werde. Wo sich aber zutragen solt oder wurde, das die haushaltung verendert und dardurch die wuchenrechnung abgeschnitten, das alsdann nichtsdesterweniger der camerschreiber sambt einem rechnungsrathe und dem rentmaister sölliche sein des cantzleiknechts ausgeben potenlons halben wochenliche rechnung empfahe und in bede wege zur zeit der hofhaltung oder verenderung derselben solliche aufgenomene und empfangne des potenmaisters rechnung fürter durch unsern rentmaister und gegenschreiber in ir rechnung unter die gebürliche tittel gebracht und verrechnet werde nach ausweisung des potenmaisters ubergeben und gethonen rechnungsregister. Und sovil des cantzleiknechts

1) Dieses Wort bedentet jedenfalls die Flachsfiden, welche zum Befestigen der Siegel verwendet wurden. Vgl. Schmeller-Frommann I, 1145. 
person geschicklichkait oder tüglichait belangt, das stellen wir euch, unsern statthaltern und räthen haimb, dermassen in dem und anderm ain aufsehen zu haben, damit durch bemelten cantzleiknecht alles zum getreulichsten verwaltet und ausgericht werde. Insonderhait ist unser mainung, das ein jeder cantzleiknecht unter anderm pflicht thue, wann er die rethe oder poten nit zu holen oder abzufertigen oder sonst in der herschaft sachen aus der rethe, cantzlers oder secretarien bevelch etwas auszurichten hab, das er alsdann nach anweisen und bevelch des cantzlers und secretarien neben andern cantzleischreibern brief oder copeien schreiben oder wess ime bevolhen wurdet, mit getreuem vleys registrirn und nit müssig geen oder in der cantzlei feirend sitzen woll. Und was euch sonst seiner pflicht halben fur gut und nothwendig ansihet, darin wöllet uns und der herschaft auch zum besten handeln." - 